\title{
Assessing eating context and fruit and vegetable consumption in children: new methods using food diaries in the UK National Diet and Nutrition Survey Rolling Programme
}

Tsz Ning Mak ${ }^{1 *}$, Celia J Prynne ${ }^{1}$, Darren Cole ${ }^{1}$, Emily Fitt $^{1}$, Caireen Roberts ${ }^{2}$, Beverley Bates ${ }^{2}$ and Alison M Stephen ${ }^{1}$

\begin{abstract}
Background: Eating context is the immediate environment of each eating occasion (EO). There is limited knowledge on the effects of the eating context on food consumption in children, due to the difficulty in measuring the multiple eating contexts children experience throughout the day. This study applied ecological momentary assessment using food diaries to explore the relationships between eating context and fruit and vegetable consumption in UK children.
\end{abstract}

Methods: Using $4 \mathrm{~d}$ unweighed food diaries, data were collected for 642 children aged 1.5-10y in two years of the UK National Diet and Nutrition Survey (2008-2010). Participants recorded all foods and drinks consumed at each EO, where and with whom the food was consumed, whether the TV was on and if eaten at a table. Mixed logistic regression and mixed multinomial logistic regression were used to calculate associations between eating contexts and fruit and vegetables (FV) consumed by quartiles.

Results: Of 16,840 EOs, $73 \%$ took place at home and 31\% with parents only. Frequency of eating alone and with friends increased with age. Compared to eating at home, children aged 1.5-3y were more likely to consume fruit at care outside home (>10-50g OR:2.39; >50-100g OR:2.12); children aged 4-6y were more likely to consume fruit (>50-100g OR:3.53; >100g OR:1.88) and vegetables at school (>30-60g OR:3.56). Compared to eating with parents only, children aged 1.5-3y were more likely to consume fruit with friends (>10-50g OR:2.69; >50-100g OR:3.49), and with carer and other children/others (>10-50g OR:2.25); children aged 4-6y were more likely to consume fruit (>50-100g OR:1.96) and vegetables with friends (>30-60g OR:3.56). Children of all ages were more likely to eat vegetables when the TV was off than on and at a table than not at table.

Conclusions: The use of food diaries to capture multiple eating contexts and detailed fruit and vegetable consumption data was demonstrated at a population level. Higher odds of FV consumption were seen from structured settings such as school and care outside home than at home, as well as when eating at a table and the TV off. This study highlights eating contexts where provision of fruit and vegetables could be improved, especially at home. Future research should take eating context into consideration when planning interventions to target children's food consumption and eating behaviour.

Keywords: Eating context, National Diet and Nutrition Survey, Fruit and vegetables, Eating occasion, Ecological Momentary Assessment, Children

\footnotetext{
* Correspondence: Tsz.Mak@mrc-hnr.cam.ac.uk

1 MRC Human Nutrition Research, Elsie Widdowson Laboratory, 120 Fulbourn

Road, CB1 9NL, Cambridge, UK

Full list of author information is available at the end of the article
} 


\section{Background}

Fruit and vegetable (FV) consumption is an established positive dietary behaviour with beneficial effects on health; consumption is linked to reduced risk of diet related diseases such as cardiovascular disease [1,2] and diabetes [3]. Children are a particular target group for promotion of increased consumption, as evidence suggests diet and eating habits in childhood are likely to track and persist into adulthood [4], and that protective properties of fruits and vegetables may need to be present in early stages of life to be effective in adulthood in preventing these diseases [5]. Thus, children who consume higher quantities of FV during childhood are likely to be healthier in later life with lower risk of developing diet-related chronic conditions [6,7].

The food environment is an important determinant of dietary behaviour. Recent studies have focussed on the availability and accessibility of FV in the built environments such as neighbourhoods $[8,9]$ and schools $[10,11]$, as well as home food environment [12-15]. However, little is known about the relationship between the immediate meal environment and FV consumption in children, particularly across multiple meal locations and social contexts. Children experience a range of social meal settings throughout the day, such as school, formal and informal care, home, as well as eating with different people under these settings. The provision of food for children is likely to be different in different locations and consumption may differ by the other people present at eating occasions. Moreover, external influences such as television and behaviours like eating at the table during meals may influence consumption. Previous studies suggest that TV watching may encourage junk food consumption [16,17] whilst eating shared family meals may encourage healthy food consumption [18]. It is therefore important to assess various eating contexts in relation to consumption in children, and identify specific contexts that may encourage or discourage positive healthy eating behaviours. This can stimulate parents and carers to consider and potentially change their children's eating environment such as to limit eating in front of the TV and to eat together at the table, as well as to enable researchers in targeting specific eating contexts in future health promotion interventions.

Measuring the eating context is difficult, particularly at a population level. Previous research has used questionnaires to measure a number of factors that describe the home environment, such as food availability, family meal and screen time [13,18-22]; such methods give indications of the usual frequency of certain exposures but not a detailed account of the context at the time of eating. A number of studies have investigated school meal surroundings and consumption using direct observation methods $[10,23]$. However, these on-site observations are only suitable for capturing single meals at specific community settings such as schools, and are likely to be burdensome and costly. A possible solution to overcome these limitations, and to capture both multiple eating contexts and to record detailed dietary data at the same time, is the use of Ecological Momentary Assessment (EMA). This approach is useful for collecting real-time data in natural environments over time and across contexts; however it has mainly been applied in physical activity research and clinical psychological studies [24,25]. To our knowledge, EMA has not been used to study the eating context at a population level in nutritional research. Our aim was to combine EMA with dietary assessment using food diaries, to study the association between eating contexts and fruit and vegetable consumption at the eating occasion level in a national representative sample of UK children. The eating context was defined by four elements: the location of the eating occasion, who was present, whether the television was on, and if consumed at a table. These four elements were selected as they were easy to record without extra burden on the participants.

\section{Methods}

\section{Study sample and design}

The data used were from 642 children aged 1.5 to 10 years from Year 1 (February 2008 to April 2009) and Year 2 (April 2009 to April 2010) of the National Diet and Nutrition Survey (NDNS) Rolling Programme. NDNS assesses food consumption, nutrient intakes and nutritional status of free living people aged 1.5 years and over in the UK. A detailed description of the sampling procedure and design of the rolling programme has been reported elsewhere [26]. The sampling is based on a random selection of postcode sectors throughout the UK, and a number of addresses per sector are visited by interviewers to invite participation, following an initial information letter. Up to one adult and one child could be selected from each participating household and a child "boost" of addresses where only children were recruited is included to ensure an even number of adults and children, since many households have no children [26]. NDNS was conducted according to the guidelines laid down in the Declaration of Helsinki and all procedures involving human subjects were approved by the Oxfordshire A Research Ethics Committee. Written informed consent was obtained from all participants [26].

\section{Dietary assessment}

At the initial interviewer visit, parents were given instructions to record all food and beverages consumed by the participating child in and out of the home over four consecutive days using unweighed food diaries. 
Recording for three days or more was considered fully productive. Parents were asked to seek help from short term carers, adult friends and relatives, and teachers with the recording process if children had been away from parents [27]. Portion sizes were estimated using household measures and using weights from food package labels obtained online or by purchase, or provided by participants. For homemade dishes, recipes were recorded separately with detailed description of ingredients, quantities and cooking methods. Further description and justification of the dietary assessment methods used in the NDNS rolling programme have been reported previously [26,27]. At the final interviewer visit, the food diaries were checked for missing information and added further detail if possible before they were returned to Medical Research Council- Human Nutrition Research for coding. The diaries were coded by trained coders using the in-house dietary assessment software, Diet In Nutrients Out (DINO), with nutrient values provided by the UK NDNS Nutrient Databank (NDB).

\section{Disaggregation of fruit and vegetable consumption}

To quantify the consumption of FV more accurately, disaggregation was undertaken to account for consumption from composite dishes containing FV, as well as discrete portions. This is particularly important in studying FV consumption in younger children, as there is a wide variety of food products intended for children e.g. toddler ready meals, snacks and dairy products such as yoghurts, that contain a mixture of fruit and/or vegetables as well as other ingredients. Portion sizes of discrete FV items were determined using FSA's reference book on Food Portion Sizes [28]. The method adopted to disaggregate food codes in NDNS has been described fully in a previous paper [29].

\section{Assessment of the eating context at meal}

In the food diaries, each recording day was divided into seven timeslots (6-9am, 9-12pm, 12-2pm, 2-5pm, 5$8 \mathrm{pm}, 8-10 \mathrm{pm}$ and $10 \mathrm{pm}-6 \mathrm{am})$ to reflect the time for various meals and snacks. For every episode of eating, parents were asked to record in the corresponding timeslot where their child was, who the child was with, whether TV was on and if the child was eating at a table, as well as the actual time of consumption (see diary extract in Additional file 1: Appendix 1). In some timeslots, children had multiple eating occasions. For each food entered into DINO, codes were allocated to describe "Where" the food was consumed, "With whom", "TV on" and "At a table". For children aged 1.5-10 years, responses for "Where" were initially coded into 29 subcategories and "With whom" coded into 12 subcategories. These subcategories were further collapsed into fewer categories to give better distribution of data (Additional file 2: Appendix 2). For children aged 1.5-3y, "Where" was condensed into "At home", "Friend's or relative's house", "Care outside home", "Other eateries", and "Other places" categories. For school children aged 4-10y, a category "At school" was added to account for eating occasions that took place within school. For children aged 7-10y, the category "Care outside home" recoded with "Other places" due to very few eating occasions taking place at care outside home in this age group. The categories for the "With whom" variable were condensed to "Parents only", "Parents and siblings", "Adult relatives and friends", "Carer and other children/others", "Alone" and "Friends". Both "Care outside home" and "Carer and other children/others" refer to formal care settings in this study. "TV on" and "Sitting at a table" were coded as "Yes" or "No". Where participants did not record answers in any of these four eating context variables, they were coded as "Not specified".

\section{Defining eating occasions}

Initially, each row of data in the dataset represented a single food item or ingredient consumed by the participant. The total number of food entries was 54,039 over the four day recording period. In order to study the relationship between the eating context at a given meal and FV consumption, food entries that were consumed together as a meal needed to be combined together. A new variable named "eating occasion" (EO) was defined, where food entries with matching answers for variables "subject ID", "time (hh:mm) of consumption", "Recording day", "where", "with whom", "TV" and "at table" were aggregated to identify the foods consumed at each EO. The number of EOs generated after aggregation was 16,830 .

\section{Potential confounders}

A number of covariates have been identified that may confound the relationship between the eating context and consumption. Consumption behaviour was likely to be different at the seven time points of the day (time slot) and between weekday and weekend days. A binary variable "weekday" was created to differentiate weekdays and weekend days, accounting for potential differences in intake due to different circumstances at weekdays when children were at school or care, versus other activities at the weekend. Children's age (years) and sex, as well as their socioeconomic status (SES) were also potential confounders. National Statistics Socio-economic Classification (NSSEC) was used as the indicator of SES in this study. NSSEC is the primary social classification used in the UK in all official statistics and surveys [30]. Therefore five covariates: children's age, sex, NSSEC, 
timeslot and weekend/weekday variation were adjusted for in the models.

\section{Statistical analysis}

Distributions of categorical variables were described in absolute frequencies and percentages. Nonparametric test for trend was used to test for associations between each category of the eating context variables and the age group (1.5-3y, 4-6y and 7-10y) of children. Fruit consumption and vegetable consumption were analysed separately in all models. Distribution of the amount consumed in both variables across EOs illustrated skewness, due to a high number of EOs where no fruit and/ or vegetables were consumed. A two part model was used to analyse the data. Firstly, outcome variables were dichotomised to differentiate EOs where fruit or vegetables were consumed (consumed $=1$; not consumed $=0$ ). Mixed-effects logistic regression was used to estimate the odds ratios (OR) of fruit consumption and vegetable consumption between the subcategories of "where" in relation to the reference subcategory. The same analyses were repeated for "with whom", "TV" and "at table" respectively. The reference subcategory for each eating context variable was: "At home" for "where"; "Parents only" for "with whom"; "Yes" for "TV on"; and "Yes" for "Eating at table".

The second part of the model assessed quantities of fruit and vegetables consumed and the four eating context variables in EOs where fruit and/vegetables were consumed, i.e. excluding all EOs with $0 \mathrm{~g}$ of consumption. EOs were divided into quartiles according to consumption level, with the lowest quartile being the reference category. For fruit, Q1: $>0-10 \mathrm{~g}, \mathrm{Q} 2:>10-50 \mathrm{~g}$, Q3: >50-100g, Q4: >100g; and for vegetables, Q1: $>0-30$ g, Q2: >30-60g, Q3: >60-100g, Q4: >100g. Multinomial mixed logistic regression was used for the analyses. The ORs calculated were compared to two reference categories. Firstly, ORs in Q2-4 were compared to the quartile reference (i.e. Q1); secondly, within each quartile, the ORs were compared to the eating context reference category (i.e. "at home" for "where", "parents only" for "with whom", "Yes" for "TV on"; and "Yes" for "Eating at table").

Mixed-effects modelling was used because each participant had repeated measures of EOs over the recording period. This method handles data where observations are not independent of each other and are clustered within group. It is therefore important to correct for this within-person variability (random effect), and the subject ID variable served as the grouping variable in the models. Complete case analyses were performed on all ages and stratified by age groups $1.5-3 y, 4-6 y$ and $7-10 y$, to take into account factors such as schooling and care as well as different stages of growth. "Not specified" responses were treated as missing. All models were adjusted for age, sex, meal time slot and weekday/weekend variations. Analyses were performed using Stata Statistical Software: Release 11 (College Station, TX: StataCorp LP). Only adjusted models are shown in this paper.

\section{Results}

\section{Characteristics of children and EOs}

Table 1 and 2 show the characteristics of participants and EOs. On average, children had four EOs per day. $36.2 \%$ of EOs took place at the weekend. There was a similar distribution of EOs in the time slots between $6 \mathrm{am}$ and $5 \mathrm{pm}$. The largest proportion of EOs occurred between $5 \mathrm{pm}-8 \mathrm{pm}$ (23.1\%), and the fewest between 8 pm-6am (8.8\%). Of the 16830 EOs, $22.2 \%$ were vegetables consuming occasions and $27.6 \%$ were fruit. Among these, the median consumption for fruit was $50.0 \mathrm{~g}$ and $59.6 \mathrm{~g}$ for vegetables. "Not specified" responses account for $2.1 \%$ in the "where" variable, $15.3 \%$ in "with whom", $21.5 \%$ in "watching TV" and $23.9 \%$ in "sitting at a table".

Distribution of EOs by where, with whom, TV and at table Over the diary recording period, children aged 1.5-3y had $80 \%$ of EOs at home and $6.6 \%$ at care outside home (Table 3). School children aged 4-10y had 69\% of EOs at home and $14 \%$ at school. Pre-schoolers ate mostly with parents only (48\%), whereas older children ate mostly with parents and siblings $(\approx 30 \%)$. As age increased, Children ate more frequently with friends (4\% 1.5-3y; 16\% 4-6y; 19\% 7-10y), and alone (4.2\% 1.5-3y; 7\% 4-6y; $12 \% 7-10 y)$. The television was off in $61 \%$ of the EOs, and children ate at the table (55\%) for the majority of the EOs. Trend tests showed significant associations between the age group of children and the four eating context variables (all $\mathrm{p}<0.05)$.

Table 1 Characteristics of children aged 1.5-10y in NDNS (2008-10)

\begin{tabular}{lrr}
\hline & $\mathbf{n}$ & Mean (S.D) \\
\hline Age & 642 & $5.3(2.8)$ \\
\hline Sex & $\mathbf{n}$ & $\mathbf{\%}$ \\
\hline Male & 327 & 50.9 \\
\hline Female & 315 & 49.1 \\
\hline Age group & & 34.1 \\
\hline $1.5-3 y$ & 219 & 29.9 \\
\hline $4-6 y$ & 192 & 36.0 \\
\hline 7-10y & 231 & Mean (S.D) \\
\hline Mean EOs per day & $\mathbf{n}$ & $4.4(2.7)$ \\
\hline $1.5-3 y$ & 219 & $4.0(2.3)$ \\
\hline 4-6y & 192 & $4.0(2.4)$ \\
\hline 7-10y & 231 & $4.1(2.5)$ \\
\hline All ages & 642 &
\end{tabular}


Table 2 Characteristics of the eating occasions

\begin{tabular}{lcr}
\hline & $\mathbf{n}$ & $\%$ \\
\hline Day of Week & 2,379 & 14.1 \\
\hline Monday & 1,822 & 10.8 \\
\hline Tuesday & 1,511 & 9.0 \\
\hline Wednesday & 2,202 & 13.1 \\
\hline Thursday & 2,815 & 16.7 \\
\hline Friday & 3,116 & 18.5 \\
\hline Saturday & 2,985 & 17.7 \\
\hline Sunday & 10,729 & 63.8 \\
\hline Weekday & 6,101 & 36.3 \\
\hline Weekend & & 15.6 \\
\hline Meal Time Slot & 2,629 & 17.5 \\
\hline 6am to 8:59am & 2,943 & 17.2 \\
\hline 9am to 11:59am & 2,889 & 17.8 \\
\hline 12pm to 1:59pm & 3,000 & 23.1 \\
\hline 2pm to 4:59pm & 3,891 & 8.8 \\
\hline 5pm to 7:59pm & 1,478 & 27.5 \\
\hline 8pm to 5:59pm & & 43.2 \\
\hline EOs consuming & 4,643 & \\
\hline Fruit & 3,734 & 7,270 \\
\hline Vegetables & & \\
\hline FV & & \\
\hline
\end{tabular}

"Where" and fruit and vegetable consumption

Compared to eating at home, children of all ages were more likely to consume fruit in EOs at school and at care outside of home, less likely in other eateries and other places (Table 4). They were more likely to consume vegetables at care outside home and at other eateries, but not at other places. When stratified by age group, children aged 1-3y had greater odds of consuming fruit and vegetables at care outside home than at home; school children (4-10y) were most likely to consume fruit at school, to consume vegetables at other eateries than at home.

In terms of the quantity consumed, compared to eating at home, children 1.5 -3y were more likely to consume between $10 \mathrm{~g}$ to $100 \mathrm{~g}(\mathrm{Q} 2$ to Q3) of fruit in EOs at care outside home (Q2 OR: 2.39; 95\% CI: 1.40-4.07; Q3 OR: 2.12; 95\% CI: 1.23-3.63) (Table 5 and Additional file 3: Figure S1). Those aged 4-6y had higher odds of consuming > 50g of fruit at school (Q3 OR: 3.53; 95\% CI: 1.95-6.37; Q4 OR: 1.88; 95\% CI: 1.06-3.32), and >30-60g of vegetables at school (OR: 3.56; 95\% CI: 1.81-7.01) than at home (Additional file 4: Figure S2). Fewer differences were seen in children aged 7-10y, they were less likely to consume $>30 \mathrm{~g}$ of vegetables at other places than at home.

Table 3 'by "Where", "With whom", “TV on", “At table”, by age groups, excluding "Not specified" responses

\begin{tabular}{|c|c|c|c|c|c|c|c|c|c|}
\hline & \multicolumn{2}{|c|}{ All ages } & \multicolumn{2}{|c|}{$1.5-3 y$} & \multicolumn{2}{|c|}{$4-6 y$} & \multicolumn{2}{|c|}{$7-10 y$} & \multirow[t]{2}{*}{ p-trend } \\
\hline & $\mathrm{n}$ & $\%$ & $\mathbf{n}$ & $\%$ & $\mathbf{n}$ & $\%$ & $\mathbf{n}$ & $\%$ & \\
\hline \multicolumn{10}{|l|}{ Where } \\
\hline At home & 12,020 & 72.9 & 4,835 & 80.3 & 3,315 & 68.6 & 3,870 & 68.8 & $<0.001$ \\
\hline At school & 1,466 & 8.9 & -—- & -— & 669 & 13.9 & 775 & 13.8 & $<0.001$ \\
\hline Friend's and relative's house & 893 & 5.4 & 299 & 5.0 & 251 & 5.2 & 343 & 6.1 & 0.007 \\
\hline Care outside home ( \& school) & 495 & 3.0 & 400 & 6.6 & 83 & 1.7 & -—- & -— & $<0.001$ \\
\hline Other eateries & 340 & 2.1 & 111 & 1.8 & 106 & 2.2 & 129 & 2.3 & 0.036 \\
\hline Other places & 1,265 & 7.7 & 376 & 6.2 & 407 & 8.4 & 510 & 9.1 & $<0.001$ \\
\hline \multicolumn{10}{|l|}{ With whom } \\
\hline Parents & 4,422 & 31.0 & 2,528 & 47.5 & 958 & 23.3 & 936 & 19.4 & $<0.001$ \\
\hline Parents and siblings & 3,816 & 26.8 & 1,051 & 19.8 & 1,308 & 31.8 & 1,457 & 30.2 & $<0.001$ \\
\hline Adult relatives and friends & 2,849 & 20.0 & 1,118 & 21.0 & 807 & 19.6 & 924 & 19.2 & 0.018 \\
\hline Alone & 1,077 & 7.6 & 222 & 4.2 & 294 & 7.1 & 561 & 11.6 & $<0.001$ \\
\hline Carer and other children/others & 358 & 2.5 & 216 & 4.1 & 95 & 2.3 & 47 & 1.0 & $<0.001$ \\
\hline Friends & 1,742 & 12.2 & 186 & 3.5 & 656 & 15.9 & 900 & 18.7 & $<0.001$ \\
\hline \multicolumn{10}{|l|}{ TV on } \\
\hline Yes & 5,217 & 39.5 & 2,139 & 43.5 & 1,412 & 37.0 & 1,666 & 37.1 & - \\
\hline No & 8,002 & 60.5 & 2,776 & 56.5 & 2,405 & 63.0 & 2,821 & 62.9 & $<0.001$ \\
\hline \multicolumn{10}{|l|}{ At table } \\
\hline Yes & 7,056 & 55.1 & 2,495 & 52.5 & 2,163 & 58.6 & 2,398 & 55.0 & - \\
\hline No & 5,748 & 44.9 & 2,254 & 47.5 & 1,530 & 41.4 & 1,964 & 45.0 & $<0.001$ \\
\hline
\end{tabular}

* Nonparametric test for trend across ordered groups was used to test if a trend existed in the distribution of each category and age group of children. 
Table 4 Odds of fruit and vegetable consumption by "where" the eating occasions took place, stratified by age group

\begin{tabular}{|c|c|c|c|c|c|c|c|c|c|c|c|c|c|c|c|c|c|c|c|c|c|c|c|c|}
\hline & \multicolumn{6}{|c|}{ All ages $(n=16479)$} & \multicolumn{6}{|c|}{$1.5-3 y(n=6021)$} & \multicolumn{6}{|c|}{$4-6 y(n=4831)$} & \multicolumn{6}{|c|}{$7-10 y(n=5627)$} \\
\hline & \multicolumn{3}{|c|}{ Fruit } & \multicolumn{3}{|c|}{ Vegetables } & \multicolumn{3}{|c|}{ Vegetables } & \multicolumn{3}{|c|}{ Vegetables } & \multicolumn{3}{|c|}{ Fruit } & \multicolumn{3}{|c|}{ Vegetables } & \multicolumn{3}{|c|}{ Fruit } & \multicolumn{3}{|c|}{ Vegetables } \\
\hline & OR & $95^{\circ}$ & $\% \mathrm{Cl}$ & OR & 95 & $6 \mathrm{Cl}$ & OR & $95 \%$ & $\% \mathrm{Cl}$ & OR & $95 \%$ & $\% \mathrm{Cl}$ & OR & $95^{\circ}$ & $6 \mathrm{Cl}$ & OR & $95^{\circ}$ & $\% \mathrm{Cl}$ & OR & $95 \%$ & $\% \mathrm{Cl}$ & OR & & $\% \mathrm{Cl}$ \\
\hline At home & 1.00 & & & 1.00 & & & 1.00 & & & 1.00 & & & 1.00 & & & 1.00 & & & 1.00 & & & 1.00 & & \\
\hline At school & 2.98 & 2.58 & 3.45 & 1.10 & 0.93 & 1.31 & - & -— & -— & -— & - - & - & 3.21 & 2.53 & 4.07 & 1.31 & 0.99 & 1.73 & 2.95 & 2.34 & 3.71 & 1.15 & 0.88 & 1.49 \\
\hline Friend's and relative's house & 0.97 & 0.81 & 1.15 & 1.10 & 0.91 & 1.32 & 1.03 & 0.77 & 1.38 & 1.19 & 0.87 & 1.62 & 0.88 & 0.63 & 1.23 & 1.26 & 0.90 & 1.77 & 0.96 & 0.71 & 1.30 & 0.92 & 0.67 & 1.25 \\
\hline Care outside home & 2.07 & 1.67 & 2.56 & 2.15 & 1.67 & 2.78 & 2.05 & 1.61 & 2.62 & 2.12 & 1.59 & 2.84 & 2.02 & 1.21 & 3.38 & 1.65 & 0.88 & 3.08 & - & - & -- & - & - - & - \\
\hline Other eateries & 0.62 & 0.47 & 0.82 & 1.99 & 1.54 & 2.56 & 0.78 & 0.50 & 1.21 & 1.44 & 0.91 & 2.27 & 0.68 & 0.42 & 1.11 & 2.36 & 1.50 & 3.72 & 0.50 & 0.29 & 0.86 & 2.15 & 1.42 & 3.26 \\
\hline Other places & 0.80 & 0.69 & 0.93 & 0.27 & 0.21 & 0.34 & 1.16 & 0.91 & 1.48 & 0.34 & 0.23 & 0.50 & 0.75 & 0.58 & 0.98 & 0.22 & 0.15 & 0.34 & 0.60 & 0.45 & 0.79 & 0.28 & 0.20 & 0.39 \\
\hline
\end{tabular}

All models adjusted for age, sex, timeslot, weekday/weekend variation and NSSEC5.

OR in bold $=p<0.05$.

(All eating occasions). 
Table 5 Odds of fruit and vegetable consumption by quartiles of amount and "where" the eating occasions took place, stratified by age group*

\begin{tabular}{|c|c|c|c|c|c|c|c|c|c|c|c|c|c|c|c|c|c|c|c|c|c|c|c|c|}
\hline & \multicolumn{6}{|c|}{ All ages } & \multicolumn{6}{|c|}{$1.5-3 y$} & \multicolumn{6}{|c|}{$4-6 y$} & \multicolumn{6}{|c|}{$7-10 y$} \\
\hline & \multicolumn{3}{|c|}{ Fruit } & \multicolumn{3}{|c|}{ Vegetables } & \multicolumn{3}{|c|}{ Fruit } & \multicolumn{3}{|c|}{ Vegetables } & \multicolumn{3}{|c|}{ Fruit } & \multicolumn{3}{|c|}{ Vegetables } & \multicolumn{3}{|c|}{ Fruit } & \multicolumn{3}{|c|}{ Vegetables } \\
\hline & \multicolumn{3}{|c|}{$n=4542$} & \multicolumn{3}{|c|}{$n=3665$} & \multicolumn{3}{|c|}{$n=1841$} & \multicolumn{3}{|c|}{$n=1259$} & \multicolumn{3}{|c|}{$\mathrm{n}=1407$} & \multicolumn{3}{|c|}{$n=1113$} & \multicolumn{3}{|c|}{$n=1294$} & \multicolumn{3}{|c|}{$n=1293$} \\
\hline & OR & \multicolumn{2}{|c|}{$95 \%$ C.I. } & OR & \multicolumn{2}{|c|}{ 95\% C.I. } & OR & \multicolumn{2}{|c|}{ 95\% C.I. } & OR & \multicolumn{2}{|c|}{ 95\% C.I. } & OR & $95 \%$ & C.I. & OR & $95 \%$ & 6 C.I. & OR & $95 \%$ & C.I. & OR & $95 \%$ & o C.I. \\
\hline At home & 1.00 & & & 1.00 & & & 1.00 & & & 1.00 & & & 1.00 & & & 1.00 & & & 1.00 & & & 1.00 & & \\
\hline At school & 1.41 & 0.99 & 2.00 & 2.20 & 1.48 & 3.28 & - & -— & -- & -- & - & -— & 1.29 & 0.71 & 2.33 & 3.56 & 1.81 & 7.01 & 1.46 & 0.79 & 2.71 & 1.90 & 0.99 & 3.64 \\
\hline Friend's and relative's house & 0.77 & 0.50 & 1.17 & 1.08 & 0.72 & 1.62 & 1.01 & 0.52 & 1.96 & 1.01 & 0.53 & 1.94 & 0.49 & 0.22 & 1.11 & 1.48 & 0.69 & 3.18 & 0.90 & 0.41 & 1.96 & 0.82 & 0.39 & 1.71 \\
\hline Care outside home & 2.35 & 1.45 & 3.81 & 1.39 & 0.83 & 2.34 & 2.39 & 1.40 & 4.07 & 1.60 & 0.89 & 2.88 & 0.98 & 0.27 & 3.51 & 0.29 & 0.03 & 2.76 & -- & -— & - & -— & -— & -二 \\
\hline Other eateries & 0.38 & 0.19 & 0.75 & 0.58 & 0.36 & 0.93 & 0.38 & 0.13 & 1.07 & 0.79 & 0.33 & 1.90 & 0.33 & 0.10 & 1.10 & 0.43 & 0.18 & 1.00 & 0.65 & 0.17 & 2.44 & 0.62 & 0.28 & 1.39 \\
\hline Other places & 0.80 & 0.54 & 1.19 & 0.36 & 0.20 & 0.64 & 0.72 & 0.41 & 1.28 & 0.19 & 0.05 & 0.70 & 0.79 & 0.40 & 1.54 & 0.30 & 0.10 & 0.94 & 0.74 & 0.29 & 1.86 & 0.41 & 0.18 & 0.95 \\
\hline Q3 & & & & & & & & & & & & & & & & & & & & & & & & \\
\hline At home & 1.00 & & & 1.00 & & & 1.00 & & & 1.00 & & & 1.00 & & & 1.00 & & & 1.00 & & & 1.00 & & \\
\hline At school & 2.55 & 1.82 & 3.58 & 1.53 & 1.01 & 2.30 & -— & -— & -— & -— & -— & -— & 3.53 & 1.95 & 6.37 & 1.38 & 0.69 & 2.77 & 1.73 & 0.95 & 3.14 & 1.91 & 1.00 & 3.65 \\
\hline Friend's and relative's house & 0.66 & 0.43 & 1.03 & 1.00 & 0.66 & 1.52 & 1.06 & 0.55 & 2.04 & 0.74 & 0.36 & 1.54 & 0.19 & 0.07 & 0.54 & 1.17 & 0.53 & 2.56 & 0.80 & 0.36 & 1.79 & 0.96 & 0.48 & 1.93 \\
\hline Care outside home & 2.15 & 1.32 & 3.50 & 1.00 & 0.57 & 1.74 & 2.12 & 1.23 & 3.63 & 0.81 & 0.41 & 1.59 & 2.33 & 0.71 & 7.60 & 1.68 & 0.49 & 5.78 & - & - & - & - & - & -— \\
\hline Other eateries & 0.37 & 0.18 & 0.74 & 0.39 & 0.23 & 0.66 & 0.60 & 0.24 & 1.53 & 0.43 & 0.15 & 1.26 & 0.37 & 0.11 & 1.26 & 0.20 & 0.07 & 0.58 & 0.43 & 0.10 & 1.86 & 0.56 & 0.25 & 1.26 \\
\hline Other places & 0.91 & 0.63 & 1.34 & 0.23 & 0.12 & 0.45 & 0.93 & 0.54 & 1.60 & 0.36 & 0.12 & 1.06 & 0.71 & 0.36 & 1.44 & 0.30 & 0.10 & 0.93 & 1.25 & 0.56 & 2.81 & 0.14 & 0.04 & 0.44 \\
\hline Q4 & & & & & & & & & & & & & & & & & & & & & & & & \\
\hline At home & 1.00 & & & 1.00 & & & 1.00 & & & 1.00 & & & 1.00 & & & 1.00 & & & 1.00 & & & 1.00 & & \\
\hline At school & 1.87 & 1.35 & 2.60 & 0.49 & 0.32 & 0.75 & - & -— & -— & - & -— & -— & 1.88 & 1.06 & 3.32 & 0.49 & 0.24 & 0.98 & 1.47 & 0.85 & 2.55 & 0.57 & 0.30 & 1.09 \\
\hline Friend's and relative's house & 0.71 & 0.47 & 1.07 & 0.63 & 0.41 & 0.96 & 1.01 & 0.51 & 2.01 & 0.52 & 0.23 & 1.16 & 0.47 & 0.22 & 1.01 & 0.88 & 0.41 & 1.91 & 0.72 & 0.35 & 1.48 & 0.50 & 0.25 & 0.99 \\
\hline Care outside home & 1.31 & 0.78 & 2.19 & 0.28 & 0.13 & 0.60 & 1.34 & 0.74 & 2.42 & 0.25 & 0.10 & 0.64 & 1.37 & 0.43 & 4.36 & 0.33 & 0.07 & 1.54 & - & -— & - - & -— & -— & -二 \\
\hline Other eateries & 0.21 & 0.09 & 0.47 & 0.25 & 0.15 & 0.43 & 0.55 & 0.19 & 1.56 & 0.22 & 0.06 & 0.80 & 0.15 & 0.03 & 0.62 & 0.20 & 0.08 & 0.49 & 0.17 & 0.03 & 0.90 & 0.32 & 0.15 & 0.70 \\
\hline Other places & 1.01 & 0.70 & 1.45 & 0.14 & 0.07 & 0.27 & 1.47 & 0.86 & 2.50 & 0.19 & 0.05 & 0.69 & 0.44 & 0.22 & 0.89 & 0.11 & 0.03 & 0.42 & 1.66 & 0.81 & 3.41 & 0.11 & 0.04 & 0.29 \\
\hline
\end{tabular}

All models adjusted for age, sex, timeslot, weekday/weekend variation and NSSEC.

OR in bold $=p<0.05$.

* In eating occasions where fruit or vegetables were consumed only. 
Table 6 Odds of fruit and vegetable consumption and eating "with whom", stratified by age group

\begin{tabular}{|c|c|c|c|c|c|c|c|c|c|c|c|c|c|c|c|c|c|c|c|c|c|c|c|c|}
\hline & \multicolumn{6}{|c|}{ All ages $(n=14264)$} & \multicolumn{6}{|c|}{$1.5-3 y(n=5321)$} & \multicolumn{6}{|c|}{$4-6 y(n=4118)$} & \multicolumn{6}{|c|}{$7-10 y(n=4825)$} \\
\hline & \multicolumn{3}{|c|}{ Fruit } & \multicolumn{3}{|c|}{ Vegetables } & \multicolumn{3}{|c|}{ Fruit } & \multicolumn{3}{|c|}{ Vegetables } & \multicolumn{3}{|c|}{ Fruit } & \multicolumn{3}{|c|}{ Vegetables } & \multicolumn{3}{|c|}{ Fruit } & \multicolumn{3}{|c|}{ Vegetables } \\
\hline & OR & \multicolumn{2}{|c|}{$95 \% \mathrm{Cl}$} & \multirow{2}{*}{$\begin{array}{l}\text { OR } \\
1.00\end{array}$} & \multicolumn{2}{|c|}{$95 \% \mathrm{Cl}$} & \multirow{2}{*}{$\begin{array}{l}\text { OR } \\
1.00 \\
\end{array}$} & \multicolumn{2}{|c|}{$95 \% \mathrm{Cl}$} & \multirow{2}{*}{$\begin{array}{l}\text { OR } \\
1.00 \\
\end{array}$} & \multicolumn{2}{|c|}{$95 \% \mathrm{Cl}$} & \multirow{2}{*}{$\begin{array}{l}\text { OR } \\
1.00 \\
\end{array}$} & \multicolumn{2}{|c|}{$95 \% \mathrm{Cl}$} & \multirow{2}{*}{$\begin{array}{l}\text { OR } \\
1.00\end{array}$} & \multicolumn{2}{|c|}{$95 \% \mathrm{Cl}$} & \multirow{2}{*}{$\begin{array}{l}\text { OR } \\
1.00 \\
\end{array}$} & \multicolumn{2}{|c|}{$95 \% \mathrm{Cl}$} & \multirow{2}{*}{$\begin{array}{l}\text { OR } \\
1.00\end{array}$} & \multicolumn{2}{|c|}{$95 \% \mathrm{Cl}$} \\
\hline & & & & & & & & & & & & & & & & & & & & & & & & \\
\hline Parent and siblings & 1.06 & 0.94 & 1.20 & 1.35 & 1.18 & 1.56 & 1.15 & 0.94 & 1.40 & 1.41 & 1.11 & 1.77 & 1.13 & 0.89 & 1.43 & 1.38 & 1.06 & 1.78 & 0.91 & 0.72 & 1.15 & 1.25 & 0.96 & 1.62 \\
\hline Adult relatives and friends & 0.99 & 0.87 & 1.13 & 1.51 & 1.32 & 1.74 & 1.02 & 0.85 & 1.23 & 1.39 & 1.13 & 1.71 & 1.00 & 0.77 & 1.29 & 1.97 & 1.51 & 2.58 & 0.91 & 0.71 & 1.17 & 1.22 & 0.94 & 1.60 \\
\hline Alone & 0.82 & 0.68 & 1.00 & 0.29 & 0.22 & 0.39 & 0.96 & 0.67 & 1.39 & 0.27 & 0.14 & 0.50 & 0.73 & 0.51 & 1.06 & 0.38 & 0.24 & 0.62 & 0.78 & 0.57 & 1.05 & 0.23 & 0.15 & 0.35 \\
\hline Carer and other children/others & 2.70 & 2.09 & 3.48 & 2.39 & 1.77 & 3.22 & 2.21 & 1.59 & 3.07 & 2.80 & 1.90 & 4.11 & 3.31 & 1.98 & 5.54 & 2.30 & 1.28 & 4.11 & 3.34 & 1.70 & 6.56 & 1.12 & 0.49 & 2.57 \\
\hline Friends & 2.14 & 1.85 & 2.48 & 1.14 & 0.96 & 1.35 & 1.80 & 1.28 & 2.53 & 1.38 & 0.90 & 2.11 & 2.30 & 1.77 & 2.99 & 1.70 & 1.25 & 2.30 & 2.01 & 1.57 & 2.58 & 0.87 & 0.66 & 1.16 \\
\hline
\end{tabular}

All models adjusted for age, sex, timeslot, weekday/weekend variation and NSSEC.

OR in bold $=p<0.05$.

(All eating occasions). 
Table 7 Odds of fruit and vegetable consumption by quartiles of amount and eating "with whom", stratified by age groups*

\begin{tabular}{|c|c|c|c|c|c|c|c|c|c|c|c|c|c|c|c|c|c|c|c|c|c|c|c|c|}
\hline & \multicolumn{6}{|c|}{ All ages } & \multicolumn{6}{|c|}{$1.5-3 y$} & \multicolumn{6}{|c|}{$4-6 y$} & \multicolumn{6}{|c|}{$7-10 y$} \\
\hline & \multicolumn{3}{|c|}{ Fruit } & \multicolumn{3}{|c|}{ Vegetables } & \multicolumn{3}{|c|}{ Fruit } & \multicolumn{3}{|c|}{ Vegetables } & \multicolumn{3}{|c|}{ Fruit } & \multicolumn{3}{|c|}{ Vegetables } & \multicolumn{3}{|c|}{ Fruit } & \multicolumn{3}{|c|}{ Vegetables } \\
\hline & \multicolumn{3}{|c|}{$n=4035$} & \multicolumn{3}{|c|}{$n=3428$} & \multicolumn{3}{|c|}{$\mathrm{n}=1678$} & \multicolumn{3}{|c|}{$n=1189$} & \multicolumn{3}{|c|}{$n=1197$} & \multicolumn{3}{|c|}{$n=1034$} & \multicolumn{3}{|c|}{$n=1160$} & \multicolumn{3}{|c|}{$n=1205$} \\
\hline & OR & \multicolumn{2}{|c|}{ 95\% C.I. } & OR & \multicolumn{2}{|c|}{ 95\% C.I. } & OR & \multicolumn{2}{|c|}{ 95\% C.I. } & OR & \multicolumn{2}{|c|}{$95 \%$ C.I. } & OR & \multicolumn{2}{|c|}{ 95\% C.I. } & OR & \multicolumn{2}{|c|}{ 95\% C.I. } & OR & \multicolumn{2}{|c|}{ 95\% C.I. } & OR & \multicolumn{2}{|c|}{ 95\% C.I. } \\
\hline & & & & & & & & & & & & & & & & & & & & & & & & \\
\hline Parents & 1.00 & & & 1.00 & & & 1.00 & & & 1.00 & & & 1.00 & & & 1.00 & & & 1.00 & & & 1.00 & & \\
\hline Parent and siblings & 1.08 & 0.81 & 1.43 & 1.09 & 0.80 & 1.47 & 0.67 & 0.44 & 1.02 & 1.01 & 0.63 & 1.61 & 1.75 & 1.00 & 3.07 & 1.48 & 0.82 & 2.69 & 1.26 & 0.68 & 2.33 & 1.13 & 0.62 & 2.05 \\
\hline Adult relatives and friends & 0.95 & 0.70 & 1.28 & 1.16 & 0.86 & 1.56 & 0.98 & 0.65 & 1.47 & 0.85 & 0.56 & 1.30 & 1.43 & 0.76 & 2.68 & 2.11 & 1.14 & 3.89 & 0.54 & 0.28 & 1.07 & 1.46 & 0.79 & 2.71 \\
\hline Alone & 1.34 & 0.80 & 2.25 & 0.66 & 0.30 & 1.42 & 2.38 & 0.85 & 6.69 & 0.29 & 0.03 & 2.79 & 1.93 & 0.68 & 5.51 & 0.86 & 0.22 & 3.29 & 0.84 & 0.36 & 1.94 & 0.86 & 0.28 & 2.63 \\
\hline Carer and other children/others & 2.57 & 1.49 & 4.43 & 1.76 & 0.98 & 3.16 & 2.25 & 1.15 & 4.42 & 1.49 & 0.72 & 3.11 & 3.42 & 0.98 & 11.94 & 4.61 & 1.28 & 16.62 & 1.59 & 0.37 & 6.79 & 0.78 & 0.11 & 5.69 \\
\hline Friends & 1.28 & 0.90 & 1.83 & 1.76 & 1.20 & 2.58 & 2.69 & 1.23 & 5.88 & 1.30 & 0.54 & 3.13 & 1.00 & 0.52 & 1.95 & 3.56 & 1.74 & 7.26 & 1.17 & 0.58 & 2.33 & 1.47 & 0.73 & 2.94 \\
\hline Q3 & & & & & & & & & & & & & & & & & & & & & & & & \\
\hline Parents & 1.00 & & & 1.00 & & & 1.00 & & & 1.00 & & & 1.00 & & & 1.00 & & & 1.00 & & & 1.00 & & \\
\hline Parent and siblings & 1.06 & 0.80 & 1.42 & 0.96 & 0.71 & 1.30 & 0.77 & 0.51 & 1.17 & 0.77 & 0.47 & 1.26 & 1.46 & 0.82 & 2.60 & 1.07 & 0.60 & 1.90 & 1.21 & 0.64 & 2.31 & 1.19 & 0.66 & 2.16 \\
\hline Adult relatives and friends & 1.02 & 0.75 & 1.39 & 0.99 & 0.73 & 1.34 & 1.11 & 0.73 & 1.68 & 0.66 & 0.43 & 1.03 & 1.09 & 0.57 & 2.10 & 1.37 & 0.75 & 2.49 & 0.85 & 0.43 & 1.66 & 1.47 & 0.80 & 2.70 \\
\hline Alone & 1.58 & 0.95 & 2.63 & 0.60 & 0.28 & 1.27 & 2.95 & 1.06 & 8.19 & 0.78 & 0.16 & 3.81 & 2.06 & 0.73 & 5.80 & 0.45 & 0.11 & 1.95 & 1.05 & 0.46 & 2.43 & 0.90 & 0.29 & 2.78 \\
\hline Carer and other children/others & 2.11 & 1.20 & 3.69 & 0.96 & 0.50 & 1.84 & 1.89 & 0.94 & 3.78 & 0.53 & 0.22 & 1.30 & 2.40 & 0.65 & 8.82 & 2.43 & 0.67 & 8.89 & 2.60 & 0.62 & 10.85 & 1.01 & 0.16 & 6.32 \\
\hline Friends & 2.18 & 1.54 & 3.09 & 1.11 & 0.74 & 1.65 & 3.49 & 1.61 & 7.57 & 0.69 & 0.26 & 1.84 & 1.96 & 1.02 & 3.75 & 1.28 & 0.61 & 2.68 & 1.99 & 0.99 & 3.98 & 1.38 & 0.69 & 2.77 \\
\hline
\end{tabular}

Q4

\begin{tabular}{|c|c|c|c|c|c|c|c|c|c|c|c|c|c|c|c|c|c|c|c|c|c|c|c|c|}
\hline Parents & 1.00 & & & 1.00 & & & 1.00 & & & 1.00 & & & 1.00 & & & 1.00 & & & 1.00 & & & 1.00 & & \\
\hline Parent and siblings & 0.95 & 0.71 & 1.26 & 0.95 & 0.71 & 1.27 & 0.67 & 0.43 & 1.04 & 0.87 & 0.54 & 1.41 & 1.25 & 0.72 & 2.19 & 0.82 & 0.48 & 1.39 & 1.04 & 0.59 & 1.84 & 1.16 & 0.69 & 1.95 \\
\hline Adult relatives and friends & 0.91 & 0.67 & 1.23 & 0.85 & 0.63 & 1.13 & 0.96 & 0.62 & 1.48 & 0.47 & 0.29 & 0.75 & 1.22 & 0.66 & 2.27 & 1.36 & 0.79 & 2.35 & 0.61 & 0.33 & 1.10 & 1.07 & 0.62 & 1.83 \\
\hline Alone & 1.17 & 0.71 & 1.92 & 0.54 & 0.27 & 1.07 & 1.80 & 0.62 & 5.24 & 0.83 & 0.17 & 4.08 & 1.86 & 0.68 & 5.11 & 0.59 & 0.18 & 1.93 & 0.78 & 0.37 & 1.63 & 0.59 & 0.22 & 1.59 \\
\hline Carer and other children/others & 1.20 & 0.67 & 2.14 & 0.47 & 0.22 & 0.99 & 0.94 & 0.44 & 2.03 & 0.38 & 0.13 & 1.12 & 1.89 & 0.54 & 6.67 & 0.37 & 0.08 & 1.80 & 0.77 & 0.18 & 3.21 & 0.67 & 0.12 & 3.73 \\
\hline Friends & 1.34 & 0.96 & 1.89 & 0.44 & 0.29 & 0.66 & 1.06 & 0.43 & 2.63 & 0.45 & 0.14 & 1.37 & 1.32 & 0.70 & 2.48 & 0.58 & 0.29 & 1.18 & 1.32 & 0.71 & 2.46 & 0.42 & 0.22 & 0.82 \\
\hline
\end{tabular}

All models adjusted for age, sex, timeslot, weekday/weekend variation and NSSEC.

OR in bold $=\mathrm{p}<0.05$.

* In eating occasions where fruit or vegetables were consumed only. 
Table 8 Odds of fruit and vegetable consumption and TV on/off, stratified by age group

\begin{tabular}{|c|c|c|c|c|c|c|c|c|c|c|c|c|c|c|c|c|}
\hline & \multicolumn{4}{|c|}{ All ages $(n=13219)$} & \multicolumn{4}{|c|}{$1.5-3 y(n=4915)$} & \multicolumn{4}{|c|}{$4-6 y(n=3817)$} & \multicolumn{4}{|c|}{$7-10 y(n=4487)$} \\
\hline & \multicolumn{2}{|r|}{ Fruit } & \multicolumn{2}{|c|}{ Vegetables } & \multicolumn{2}{|r|}{ Fruit } & \multicolumn{2}{|c|}{ Vegetables } & \multicolumn{2}{|r|}{ Fruit } & \multicolumn{2}{|c|}{ Vegetables } & \multicolumn{2}{|r|}{ Fruit } & \multicolumn{2}{|c|}{ Vegetables } \\
\hline & OR & $95 \% \mathrm{Cl}$ & OR & $95 \% \mathrm{Cl}$ & OR & $95 \% \mathrm{Cl}$ & OR & $95 \% \mathrm{Cl}$ & OR & $95 \% \mathrm{Cl}$ & OR & $95 \% \mathrm{Cl}$ & OR & $95 \% \mathrm{Cl}$ & OR & $95 \% \mathrm{Cl}$ \\
\hline TV on & 1.00 & & 1.00 & & 1.00 & & 1.00 & & 1.00 & & 1.00 & & 1.00 & & 1.00 & \\
\hline TV off & 0.98 & $\begin{array}{ll}0.89 & 1.08\end{array}$ & 1.51 & $1.35 \quad 1.68$ & 0.94 & $\begin{array}{ll}0.81 & 1.09\end{array}$ & 1.63 & $1.36 \quad 1.95$ & 1.03 & $0.87 \quad 1.23$ & 1.53 & $1.24 \quad 1.88$ & 1.00 & $0.84 \quad 1.19$ & 1.47 & 1.221 .77 \\
\hline
\end{tabular}

All models adjusted for age, sex, timeslot, weekday/weekend variation and NSSEC.

OR in bold $=p<0.05$.

(All eating occasions).

\section{"With whom" and fruit and vegetable consumption}

Compared to eating with parents only, children of all ages were more likely to eat both fruit and vegetables with carer and other children/others; more likely to consume fruit with friends; to consume vegetables with parents and siblings and adult family and friends; but least likely to eat vegetables when alone (Table 6). When stratified by age group, the same patterns were found for children aged 1.5-3y and 4-6y, except that those aged 4$6 y$ were also more likely to consume vegetables with friends than with parents. Fewer significant associations were found for children aged 7-10y. They were more likely to eat fruit with friends, but less likely to eat vegetables alone.

Pre-schoolers (1.5-3y) had higher odds of consuming between >10-100g (Q2 and Q3) of fruit with friends (Q2 OR: 2.69; 95\% CI: 1.23-5.88; Q3 OR: 3.49; 95\% CI: 1.617.57 ), consuming between $>10-50 \mathrm{~g}$ of fruit with a carer and other children/others (OR: 2.25; 95\% CI: 1.15-4.42), and to eat $>50-100 \mathrm{~g}$ of fruit alone (OR: $2.95 ; 95 \% \mathrm{CI}$ : 1.06-8.19) than with parents only (Table 7). On the other hand, they were less likely to consume $>100 \mathrm{~g}$ of vegetables with adult relatives and friends than with parents (OR: 0.47; 95\% CI: 0.29-0.75). Children 4-6y had a greater odds of consuming $>30-60 \mathrm{~g}$ of vegetables with carer and other children/others (OR: 4.61; 95\% CI: 1.2816.62) and with adult relatives and friends (OR: 2.11; 95\% CI: $1.14-3.89$ ) and $>50-100 \mathrm{~g}$ of fruit and $>30-60 \mathrm{~g}$ of vegetables with friends (OR: 1.96; 95\% CI: 1.02-3.75 and OR: 3.56 ; 95\% CI: $1.74-7.26$ respectively) than with parents. No significant associations were found for children aged $7-10 y$, except that they were less likely to eat $>100 \mathrm{~g}$ of vegetables with friends than with parents $(\mathrm{OR}=0.42$; 95\% CI: 0.22, 0.82).

\section{"TV on" and fruit and vegetable consumption}

Across all ages, as well as by stratum, children were more likely to eat vegetables when the TV was off (Table 8). Few associations were found between the quantity consumed and the TV on or off, except for weak associations with consuming $>100 \mathrm{~g}$ of fruit in children 1.5-3y and 4-6y (data not shown).

\section{"Eating at a table" and fruit and vegetable consumption}

In terms of eating at a table, children aged 1.5-3y and 4$6 y$ were less likely to consume fruit or vegetables when they were not eating at a table (Table 9). The associations were stronger for vegetables than for fruit. For children aged 7-10y, no difference was found for fruit; however they were less likely to consume vegetables when not eating at a table.

When quantifying fruit and vegetable consumption at the table, opposite trends were seen between fruit and vegetables. Children were more likely to consume large amounts $(>100 \mathrm{~g})$ of fruit when not eating at a table (Table 10). Those aged 7-10y had significantly higher odds of consuming $>10 \mathrm{~g}(\mathrm{Q} 2, \mathrm{Q} 3$ and Q4) of fruit when not eating at a table (Q2 OR: 1.75; 95\% CI: 1.06-2.87; Q3 OR: 1.73; 95\% CI: 1.07-2.82; Q4 OR: 2.01; 95\% CI: 1.29 $3.13)$. On the contrary, not eating at a table greatly reduced the odds of consuming all amounts of vegetables in children, especially in the 7-10y age group.

Table 9 Odds of fruit and vegetable consumption and "eating at table", stratified by age group

\begin{tabular}{|c|c|c|c|c|c|c|c|c|c|c|c|c|c|c|c|c|c|c|c|c|c|c|}
\hline & \multicolumn{6}{|c|}{ All ages $(n=12804)$} & \multicolumn{6}{|c|}{$1.5-3 y(n=4749)$} & \multicolumn{6}{|c|}{$4-6 y(n=3493)$} & \multicolumn{4}{|c|}{$7-10 y(n=4362)$} \\
\hline & \multicolumn{3}{|c|}{ Fruit } & \multicolumn{3}{|c|}{ Vegetables } & \multicolumn{3}{|c|}{ Fruit } & \multicolumn{3}{|c|}{ Vegetables } & \multicolumn{3}{|c|}{ Fruit } & \multicolumn{3}{|c|}{ Vegetables } & \multicolumn{2}{|r|}{ Fruit } & \multicolumn{2}{|c|}{ Vegetables } \\
\hline & OR & $95 \%$ & $\% \mathrm{Cl}$ & OR & & $\% \mathrm{Cl}$ & OR & $95 \%$ & $\% \mathrm{Cl}$ & OR & & $\% \mathrm{Cl}$ & OR & & $\% \mathrm{Cl}$ & OR & $95 \%$ & $\% \mathrm{Cl}$ & OR & $95 \% \mathrm{Cl}$ & OR & $95 \% \mathrm{Cl}$ \\
\hline $\begin{array}{l}\text { At } \\
\text { table }\end{array}$ & 1.00 & & & 1.00 & & & 1.00 & & & 1.00 & & & 1.00 & & & 1.00 & & & 1.00 & & 1.00 & \\
\hline $\begin{array}{l}\text { Not } \\
\text { at } \\
\text { table }\end{array}$ & 0.69 & 0.63 & 0.76 & 0.11 & 0.10 & 0.13 & 0.58 & 0.50 & 0.66 & 0.10 & 0.08 & 0.12 & 0.70 & 0.59 & 90.83 & 0.16 & 0.13 & 0.20 & 0.92 & $0.78 \quad 1.09$ & 0.09 & $0.07 \quad 0.12$ \\
\hline
\end{tabular}

All models adjusted for age, sex, timeslot, weekday/weekend variation and NSSEC.

OR in bold $=p<0.05$.

(All eating occasions). 
Table 10 Odds of fruit and vegetable consumption by quartiles of amount and "eating at table", stratified by age group*

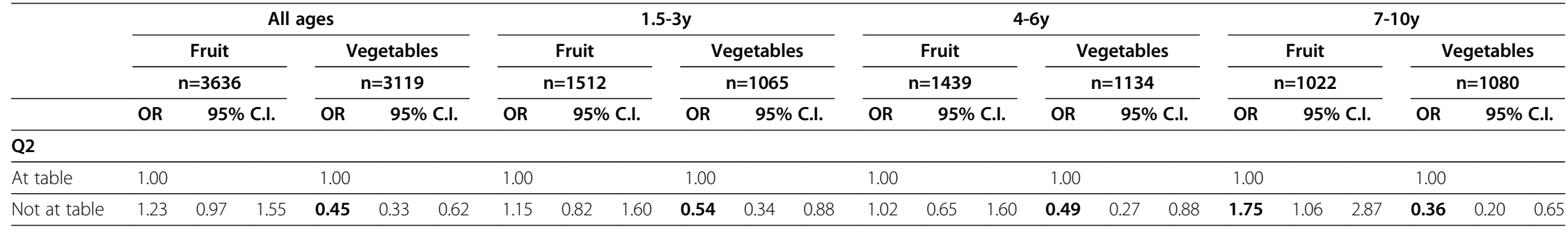

Q3

\begin{tabular}{|c|c|c|c|c|c|c|c|c|c|c|c|c|c|c|c|c|c|c|c|c|c|c|c|c|}
\hline At table & 1.00 & & & 1.00 & & & 1.00 & & & 1.00 & & & 1.00 & & & 1.00 & & & 1.00 & & & 1.00 & & \\
\hline Not at table & 1.42 & 1.13 & 1.79 & 0.65 & 0.48 & 0.87 & 1.37 & 0.98 & 1.91 & 0.63 & 0.39 & 1.04 & 1.29 & 0.83 & 2.01 & 1.02 & 0.61 & 1.73 & 1.73 & 1.07 & 2.82 & 0.40 & 0.22 & 0.71 \\
\hline
\end{tabular}

\begin{tabular}{|c|c|c|c|c|c|c|c|c|c|c|c|c|c|c|c|c|c|c|c|c|c|c|c|c|}
\hline At table & 1.00 & & & 1.00 & & & 1.00 & & & 1.00 & & & 1.00 & & & 1.00 & & & 1.00 & & & 1.00 & & \\
\hline Not at table & 1.70 & 1.36 & 2.14 & 0.44 & 0.33 & 0.60 & 1.60 & 1.13 & 2.27 & 0.43 & 0.25 & 0.75 & 1.73 & 1.13 & 2.64 & 0.69 & 0.41 & 1.17 & 2.01 & 1.29 & 3.13 & 0.27 & 0.16 & 0.46 \\
\hline
\end{tabular}

All models adjusted for age, sex, timeslot, weekday/weekend variation and NSSEC.

OR in bold $=p<0.05$.

* In eating occasions where fruit or vegetables were consumed only. 


\section{Discussion}

In this study we demonstrated a new methodology for measuring the eating context of young children and related it to fruit and vegetable consumption in a nationally representative sample of UK children. The results provide new insights into how fruit and/or vegetable consumption varies in different eating locations, with different people present, whether or not the TV is on and if eaten at a table. The results from this sample of children suggested that they were more likely to consume higher amounts of fruit and vegetables in structured and formal care settings, such as school and care outside of home than at home, and with carers and other children and their friends, than compared to the EOs taking place with parents alone. These associations were particularly strong for the younger age groups (1.5-3y and 4-6y). Fewer significant associations were seen for older children.

There are a number of possible explanations for the higher fruit and vegetable consumption seen in structured care and school settings. In 2005, the Department of Education and Skills produced guidelines to implement nutrition standards for lunches in nursery schools, which included a recommendation that an item of fruit and vegetables to be provided to each child at lunchtime each day [31]. The Caroline Walker Trust published nutrient-based standards for children under 5 years in child care, which recommended that carers offer 4 to 5 different types of fruit and vegetables to children each day in day care settings [32]. For school children, the School Meals Review Panel was appointed by the UK government in 2005 to introduce new standards for school food, whereby schools had to provide at least one portion of fruit and one portion of vegetables or salad at lunch per pupil per day [33]. Furthermore, children aged 4-6y in England who attend local education authority (LEA) maintained schools are entitled to a free piece of fruit or vegetables each school day [34], which may explain the higher odds ratios for fruit (Q3) and vegetables (Q2) in this age group, which were not seen in the 7-10y age group. These changes in recommendations may well explain the increased consumption in school and formal care settings.

Older children (7-10y) had a greater tendency to eat with friends as well as alone compared to younger children, thus illustrating the gain in independence and the increasing importance of peers in eating and food choice as they become older [35,36]. A number of intervention studies have shown that children have increased consumption of, or have expressed a liking of fruit and vegetables, due to the influence of their peers eating or liking fruit and vegetables [37-39]. Our observational study showed that children were consuming more fruit and vegetables when they were with friends than with parents, suggesting a possible effect of peer modelling on consumption of healthy foods. However, higher consumption with friends could also be confounded by the location of the eating occasions such as school or care settings. This is a speculation since we did not test the combined effects of the eating contexts on consumption. Having the TV on appeared to influence the odds of consuming vegetables, but was not associated with the quantity consumed. No strong associations were seen for fruit. Our findings only partially agree with previous studies, where fruit and vegetable consumption has been found to be inversely correlated with the frequency or number of hours of TV watching [22,40,41]. Our results are based on data for each eating occasion and may therefore capture differences in behaviour between fruit consumption and vegetable consumption, which may not be seen using questionnaires as in the other studies of the influence of television.

This study also exhibited new information on eating at the table and fruit and vegetable consumption, since this determinant has not been well researched. Children who ate at the table were much more likely to eat vegetables. This was not surprising, since previous studies have shown that family meal frequency is important in establishing positive eating behaviours such as eating fruit and vegetables, and family meals are more likely to occur around a table [42-45]. However, interesting findings were seen when quantifying the amount consumed when eating at the table. While the first part of the model suggested that children were more likely to consume fruit at the table, the second part of the model indicated large portions of fruit $(>100 \mathrm{~g})$ were consumed when not at a table. We are unsure of the reason for this observation, but a possible explanation could be that the higher odds of fruit consumption in the first part of the model were driven by higher frequency of occasions consuming small amounts of fruit in composite dishes (such as fruit puddings and desserts) at the table; whilst results in the second part of the model might have been driven by fruit in large discrete portions, such as whole apples and bananas, with typical portion sizes of around 100g, that were likely to be eaten on the go and in any environment without being at the table. Unlike fruit, vegetables were less likely to be consumed in large quantities when not at the table. This is because vegetables are usually served as part of a main meal in substantial portions rather than eaten as snacks, and hence vegetables are more likely to be eaten at a table. These findings emphasise that eating fruit and eating vegetables are different behaviours.

There are a number of strengths of this study. It illustrates an alternative methodology for assessing the eating environment using a simple traditional dietary assessment tool - a food diary. The advantage of this method is that it is easy to use, and collects real time information without using advanced technology, which 
remains important for national surveys where there are different levels of technology access and expertise and the wide ranges of age and sociodemographic background among participants. The burden of collecting the information is also low, since it can be combined as part of an existing dietary assessment tool (food diary or 24 hour recall) without using a separate questionnaire or other psychometric measure. Previously, data on the food environment, such as television watching and screen time and family meal frequency have been collected via questionnaires, while dietary intake was assessed using separate dietary assessment method. Our method is likely to be more accurate since we have detailed dietary data that match with the eating contexts for each eating occasion. Other studies had investigated specific meals such as school lunches using direct observation methods; the method described here dispenses with interviewer burden as well as the discomfort for participants of being watched and monitored.

This study has its limitations however. Children's fruit and vegetable consumption and the eating context information were reported by their parents. Previous studies have shown low agreements between children and parental reports of fruit and vegetable intakes [46-48], and also parents had to rely on information given by other carers if the children had been away from home. Hence, there could be errors induced during the recording process. Secondly, there were a number of "Not specified" responses when collecting information on the four eating context elements, particularly in answering whether the TV was on, and eating at the table. This may be because the children were in eating contexts where televisions or tables were not present, for instance, in outdoor areas or when eating on the go. These "Not specified" responses were treated as missing data because it would be bias to make assumptions on these data. The fact that only complete case analyses were performed may have resulted in reduced statistical power to detect differences amongst categories, as the number of eating occasions included in the analyses was lowered. Another limitation was that parents were only asked to record if TV was on at the occasion, it was unknown whether the children were actively watching TV during the meal and how much distraction from the TV might have influenced consumption. Moreover, NDNS did not collect data on other sociodemographic measures such as parental education that might have affected consumption. These factors were not adjusted for in the analysis and hence, there could be residual confounding in the results.

Furthermore, this study could not determine the direction of the effects between the eating context and fruit and vegetable consumption and if the effects were causal, because of the cross sectional design of the
NDNS survey. For instance, it is possible that fruit and vegetable consumption is driven by the eating contexts, such as the location of the meal, but there may also be circumstances where eating contexts are driven by food choices or consumption. Further research should therefore be done longitudinally to allow clearer causality conclusions to be drawn. In addition, this new method of eating context assessment needs validation of its accuracy as we have only focused on one food group as dietary outcome, it should also be re-tested on other populations and sub-populations, as well as on other dietary assessment tools such as 24 hour recall. Relationships between the eating context and other dietary outcomes such as energy dense food and drinks should be studied as they may also be related to the eating environment. We intend to apply this assessment method to Year 3 and 4 of the rolling programme for validity as well as testing it on other age groups and food groups. Lastly, the four eating context elements in this study have been analysed separately and this limits the understanding the relative weight of each element and the combined effect of the eating contexts on overall consumption. Hence, for future work, these factors could be modelled in such a way that their association with the outcome as well as with one another can be determined.

\section{Conclusions}

This study illustrated a new way of capturing and assessing various eating contexts using current dietary assessment tools at the population level. In this sample population, we found that children were more likely to consume fruit and vegetables at structured and regulated settings, such as schools and formal child care than at home, as well as eating at a table and with TV off. The results may encourage parents and practitioners to create and promote positive social eating environments and to provide better food choices for children, particularly at home. Researchers should also consider the eating contexts when designing future programmes and interventions that target dietary behaviours and food consumption.

\section{Additional files}

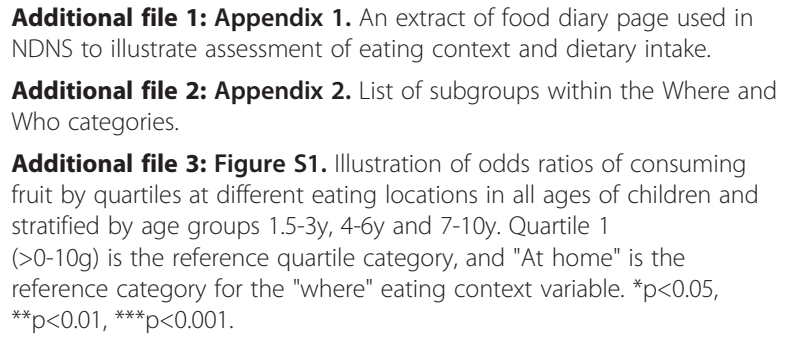

Additional file 3: Figure S1. Illustration of odds ratios of consuming fruit by quartiles at different eating locations in all ages of children and stratified by age groups 1.5-3y, 4-6y and 7-10y. Quartile 1

$(>0-10 \mathrm{~g})$ is the reference quartile category, and "At home" is the reference category for the "where" eating context variable. ${ }^{*} p<0.05$, ${ }^{* *} \mathrm{p}<0.01$ ， ${ }^{* * *} \mathrm{p}<0.001$. 
Additional file 4: Figure S2. Illustration of odds ratios of consuming vegetables by quartiles at different eating locations in all ages of children and stratified by age groups 1.5-3y, 4-6y and 7-10y. Quartile $1(>0-30 \mathrm{~g})$ is the reference quartile category, and "At home" is the reference category for the "where" eating context variable. ${ }^{*} p<0.05,{ }^{* *} p<0.01,{ }^{* * *} p<0.001$.

\section{Abbreviations}

Cl: Confidence interval; DINO: Diet in nutrients out; EOs: Eating occasions; EMA: Ecological momentary Aassessment; FSA: Food standards agency; FV: Fruit and vegetables; LEA: Local education authority; NDB: Nutrient databank; NDNS: National diet and nutrition survey; NSSEC: National statistics socio-economic classification; OR: Odds ratio; SES: Socio-economic status.

\section{Competing interests}

The authors declare no competing interests.

\section{Authors' contributions}

TNM developed the methodology, analysed and interpreted the data, and wrote the manuscript. CJP and TNM prepared the dietary data for analysis. TNM prepared the eating context data. AMS initiated the study idea, oversaw the NDNS dietary data collection, coding and analysis, supervised and edited the manuscript. All authors read and approved the final manuscript.

\section{Acknowledgements}

The National Diet and Nutrition Survey is funded by the Department of Health and Food Standards Agency

This work was supported by the UK Medical Research Council

TNM is in receipt of PhD funding support from Cambridge Overseas Trust The authors would like to thank the study participants, the NDNS team and particularly the dietary assessment assistants at MRC Human Nutrition Research who coded the food diaries

\section{Author details}

${ }^{1}$ MRC Human Nutrition Research, Elsie Widdowson Laboratory, 120 Fulbourn Road, CB1 9NL, Cambridge, UK. ${ }^{2}$ National Centre for Social Research, 35 Northampton Square, London ECIV OAX, UK.

Received: 31 January 2012 Accepted: 25 September 2012 Published: 18 October 2012

\section{References}

1. Liu S, Manson JE, Lee IM, Cole SR, Hennekens CH, Willett WC, Buring JE: Fruit and vegetable intake and risk of cardiovascular disease: the Women's Health Study. Am J Clin Nutr 2000, 72:922-928.

2. Crowe FL, Roddam AW, Key TJ, Appleby PN, Overvad K, Jakobsen MU, Tjønneland A, Hansen L, Boeing H, Weikert C, et al: Fruit and vegetable intake and mortality from ischaemic heart disease: results from the European Prospective Investigation into Cancer and Nutrition (EPIC)Heart study. Eur Heart J 2011, 32:1235-1243.

3. Harding AH, Wareham NJ, Bingham SA, Khaw K, Luben R, Welch A, Forouhi NG: Plasma vitamin C level, fruit and vegetable consumption, and the risk of new-onset type 2 diabetes mellitus: the European prospective investigation of cancer-Norfolk prospective study. Arch Intern Med 2008, 168:1493-1499.

4. Beauchamp GK, Mennella JA: Early flavor learning and its impact on later feeding behavior. J Pediatr Gastroenterol Nutr 2009, 48(Suppl 1):S25-S30

5. Willett WC: Fruits, vegetables, and cancer prevention: turmoil in the produce section. J Natl Cancer Inst 2010, 102:510-511.

6. Maynard M, Gunnell D, Emmett P, Frankel S, Davey Smith G: Fruit, vegetables, and antioxidants in childhood and risk of adult cancer: the Boyd Orr cohort. J Epidemiol Community Health 2003, 57:218-225.

7. Mikkila V, Rasanen L, Raitakari OT, Pietinen P, Viikari J: Longitudinal changes in diet from childhood into adulthood with respect to risk of cardiovascular diseases: The Cardiovascular Risk in Young Finns Study. Eur J Clin Nutr 2004, 58:1038-1045.

8. Timperio A, Ball K, Roberts R, Campbell K, Andrianopoulos N, Crawford D: Children's Fruit and vegetable intake: associations with the neighbourhood food environment. Prev Med 2008, 46:331-335.
9. Skidmore P, Welch A, van Sluijs E, Jones A, Harvey I, Harrison F, Griffin S, Cassidy A: Impact of neighbourhood food environment on food consumption in children aged 9-10 years in the UK SPEEDY (Sport, Physical Activity and Eating behaviour: Environmental Determinants in Young people) study. Public Health Nutr 2010, 13:1022-1030.

10. Briefel RR, Crepinsek MK, Cabili C, Wilson A, Gleason PM: School food environments and practices affect dietary behaviors of US public school children. J Am Diet Assoc 2009, 109:S91-S107.

11. Harrison F, Jennings A, Jones A, Welch A, van Sluijs E, Griffin S, Cassidy A: Food and drink consumption at school lunchtime: the impact of lunch type and contribution to overall intake in British 9-10-year-old children. Public Health Nutr 2011, 1-8. Epub ahead of print.

12. Ding D, Sallis JF, Norman GJ, Saelens BE, Harris SK, Kerr J, Rosenberg D, Durant N, Glanz K: Community food environment, home food environment, and fruit and vegetable intake of children and adolescents. J Nutr Educ Behav 2011, [Epub ahead of print].

13. Vereecken C, Haerens L, De Bourdeaudhuij I, Maes L: The relationship between children's home food environment and dietary patterns in childhood and adolescence. Public Health Nutr 2010, 13:1729-1735.

14. Campbell KJ, Crawford DA, Salmon J, Carver A, Garnett SP, Baur LA: Associations between the home food environment and obesity-promoting eating behaviors in adolescence. Obesity 2007, 15:719-730.

15. Arcan C, Neumark-Sztainer D, Hannan P, van den Berg P, Story M, Larson N: Parental eating behaviours, home food environment and adolescent intakes of fruits, vegetables and dairy foods: longitudinal findings from Project EAT. Public Health Nutr 2007, 10:1257-1265.

16. Matheson DM, Killen JD, Wang Y, Varady A, Robinson TN: Children's food consumption during television viewing. Am J Clin Nutr 2004, 79:1088-1094.

17. Miller SA, Taveras EM, Rifas-Shiman SL, Gillman MW: Association between television viewing and poor diet quality in young children. Int J Pediatr Obes 2008, 3:168-176.

18. Hammons $\mathrm{AJ}$, Fiese $\mathrm{BH}$ : Is frequency of shared family meals related to the nutritional health of children and adolescents? Pediatrics 2011, 127:e1565-e1574.

19. Sandvik C, De Bourdeaudhuij I, Due P, Brug J, Wind M, Bere E, Perez-Rodrigo C, Wolf A, Elmadfa I, Thorsdottir I, et al: Personal, social and environmental factors regarding fruit and vegetable intake among schoolchildren in nine European countries. Ann Nutr Metab 2005, 49:255-266.

20. Reinaerts $E$, de Nooijer J, Candel M, de Vries N: Explaining school children's fruit and vegetable consumption: the contributions of availability, accessibility, exposure, parental consumption and habit in addition to psychosocial factors. Appetite 2007, 48:248-258.

21. Bauer K, Neumark-Sztainer D, Fulkerson J, Hannan P, Story M: Familial correlates of adolescent girls' physical activity, television use, dietary intake, weight, and body composition. Int J Behav Nutr Phys Act 2011, 8:25.

22. Boutelle KN, Birnbaum AS, Lytle LA, Murray DM, Story M: Associations between perceived family meal environment and parent intake of fruit, vegetables, and fat. J Nutr Educ Behav 2003, 35:24-29.

23. Woodruff SJ, Hanning RM, McGoldrick K: The influence of physical and social contexts of eating on lunch-time food intake among southern Ontario, Canada, middle school students. J Sch Health 2010, 80:421-428.

24. Dunton GF, Liao Y, Intille S, Wolch J, Pentz MA: Physical and social contextual influences on children's leisure-time physical activity: an ecological momentary assessment study. J Phys Act Health 2011, 8(Suppl 1):S103-108.

25. Shiffman S, Stone AA, Hufford MR: Ecological momentary assessment. Ann Rev Clin Psych 2008, 4:1-32.

26. National Diet and Nutrition Survey: Headline results from Years 1 and 2 (combined) of the rolling programme 2008/9 - 2009/10; http://www.dh.gov. uk/prod_consum_dh/groups/dh_digitalassets/documents/digitalasset/ dh_128550.pdf.

27. National Diet and Nutrition Survey: Headline results from Years 1 and 2 (combined) of the rolling programme 2008/9 - 2009/10. Appendix A. Dietary data collection and editing; http://www.dh.gov.uk/prod_consum_dh/groups/ dh_digitalassets/documents/digitalasset/dh_128546.pdf.

28. Food Standards Agency: Food portion sizes. 3rd edition. London: The Stationery Office; 2002.

29. Fitt E, Mak TN, Stephen AM, Prynne C, Roberts C, Swan G, Farron-Wilson M: Disaggregating composite food codes in the UK National Diet and Nutrition Survey food composition databank. Eur J Clin Nutr 2010, 64:\$32-\$36. 
30. Rose D, Pevalin DJ, O'Reilly K: The National Statistics Socio-economic Classification: Origins, Development and Use. In Book The National Statistics Socio-economic Classification: Origins, Development and Use:: City: Institute for Social and Economic Research, University of Essex; 2005.

31. Laying the Table. Recommendations for National Food and Nutrition Guidance for Early Years Settings in England. Volume 1: Main report. http://www.schoolfoodtrust.org.uk/download/documents/pdf/ sft_laying_the_table_early_years_volume1.pdf.

32. Eating well for under $5 s$ in child care. Practical and nutritional guidelines. 2nd edition. http://www.cwt.org.uk/pdfs/Under5s.pdf.

33. School meal provision in England and other Western countries: a review. http://www.schoolfoodtrust.org.uk/download/documents/pdf/ sft_school_meals_review_may07.pdf.

34. School Fruit and Vegetable Scheme. http://www.dh.gov.uk/en/Publichealth/ Healthimprovement/FiveADay/FiveADaygeneralinformation/DH_4002149.

35. Cutler GJ, Flood A, Hannan P, Neumark-Sztainer D: Multiple sociodemographic and socioenvironmental characteristics are correlated with major patterns of dietary intake in adolescents. J Am Diet Assoc 2011, 111:230-240.

36. Story M, Neumark-Sztainer D, French S: Individual and environmental influences on adolescent eating behaviors. J Am Diet Assoc 2002, 102:S40-S51.

37. Lowe CF, Horne PJ, Tapper K, Bowdery M, Egerton C: Effects of a peer modelling and rewards-based intervention to increase fruit and vegetable consumption in children. Eur J Clin Nutr 2004, 58:510-522.

38. Horne PJ, Tapper K, Lowe CF, Hardman CA, Jackson MC, Woolner J: Increasing children's fruit and vegetable consumption: a peer-modelling and rewards-based intervention. Eur J Clin Nutr 2004, 58:1649-1660.

39. Cullen KW, Baranowski T, Rittenberry L, Cosart C, Hebert D, de Moor C: Child-reported family and peer influences on fruit, juice and vegetable consumption: reliability and validity of measures. Health Educ Res 2001, 16:187-200

40. Boynton-Jarrett R, Thomas TN, Peterson KE, Wiecha J, Sobol AM, Gortmaker SL: Impact of television viewing patterns on fruit and vegetable consumption among adolescents. Pediatrics 2003, 112:1321-1326.

41. FitzPatrick E, Edmunds LS, Dennison BA: Positive effects of family dinner Are undone by television viewing. J Am Diet Assoc 2007, 107:666-671.

42. Videon TM, Manning CK: Influences on adolescent eating patterns: the importance of family meals. J Adolesc Health 2003, 32:365-373.

43. Andaya AA, Arredondo EM, Alcaraz JE, Lindsay SP, Elder JP: The association between family meals, TV viewing during meals, and fruit, vegetables, soda, and chips intake among Latino children. J Nutr Educ Behav 2011, 43:308-315.

44. Neumark-Sztainer D, Wall M, Perry C, Story M: Correlates of fruit and vegetable intake among adolescents: findings from project EAT. Prev Med 2003, 37:198-208.

45. Neumark-Sztainer D, Hannan PJ, Story M, Croll J, Perry C: Family meal patterns: associations with sociodemographic characteristics and improved dietary intake among adolescents. J Am Diet Assoc 2003, 103:317-322.

46. Tak NI, te Velde SJ, de Vries JH, Brug J: Parent and child reports of fruit and vegetable intakes and related family environmental factors show low levels of agreement. J Hum Nutr Diet 2006, 19:275-285.

47. Bere E, Klepp K-I: Correlates of fruit and vegetable intake among Norwegian schoolchildren: parental and self-reports. Public Health Nutr 2004, 7:991-998.

48. Reinaerts E, de Nooijer J, de Vries NK: Parental versus child reporting of fruit and vegetable consumption. Int J Behav Nutr Phys Act 2007, 4:33.

doi:10.1186/1479-5868-9-126

Cite this article as: Mak et al: Assessing eating context and fruit and vegetable consumption in children: new methods using food diaries in the UK National Diet and Nutrition Survey Rolling Programme.

International Journal of Behavioral Nutrition and Physical Activity 2012 9:126.

\section{Submit your next manuscript to BioMed Central and take full advantage of:}

- Convenient online submission

- Thorough peer review

- No space constraints or color figure charges

- Immediate publication on acceptance

- Inclusion in PubMed, CAS, Scopus and Google Scholar

- Research which is freely available for redistribution

Submit your manuscript at www.biomedcentral.com/submit
C Biomed Central 